\section{Case Reports in Ophthalmology}

Case Rep Ophthalmol 2020;11:507-515

DOI: $10.1159 / 000509861$

Published online: September 29, 2020
(C) 2020 The Author(s)

Published by S. Karger AG, Basel www.karger.com/cop

This article is licensed under the Creative Commons Attribution-NonCommercial 4.0 International License (CC BY-NC) (http://www.karger.com/Services/OpenAccessLicense). Usage and distribution for commercial purposes requires written permission.

\title{
Paraneoplastic Focal Outer Retinitis and Optic Neuropathy in a Patient with Small Cell Lung Carcinoma and Anti-CRMP5, Anti-HU and Anti-Amphiphysin Antibodies
}

\author{
Clara Martínez-Rubio ${ }^{a}$ Lena Giralt ${ }^{b}$ Inazio Lacambra ${ }^{c}$ Joseba Artaraz ${ }^{b}$ \\ Alex Fonollosa ${ }^{b}$ \\ aDepartment of Ophthalmology, La Fe University and Polytechnic Hospital, University of \\ Valencia, Valencia, Spain; ${ }^{b}$ Department of Ophthalmology, BioCruces Bizkaia Health \\ Research Institute, Cruces University Hospital, University of the Basque Country, \\ Barakaldo, Spain; 'Department of Oncology, BioCruces Bizkaia Health Research Institute, \\ Cruces University Hospital, University of the Basque Country, Barakaldo, Spain
}

\section{Keywords}

Retinitis - Optic disc oedema - Ataxia - Collapsin response mediator protein 5 - Paraneoplastic syndrome

\begin{abstract}
Our aim is to describe clinical and optical coherence tomographic features of acute paraneoplastic focal outer retinitis associated with optic neuropathy in a patient diagnosed with small cell lung carcinoma. Bilateral focal outer retinitis, bilateral optic disc oedema and vitritis were identified in a patient with progressive bilateral visual loss and ataxia. Spectral domain optical coherence tomography (SD-OCT) revealed various extents of granular hyperreflectivity and atrophy of the macular outer retinal layers. Serum and cerebrospinal fluid positivity for antiCRMP5, anti-HU and anti-amphiphysin antibodies intensified the search for an underlying malignancy, and a small cell lung carcinoma (SCLC) was finally diagnosed. Local and oral steroids along with systemic chemotherapy and radiotherapy achieved cancer remission and partial recovery from the ophthalmic manifestations. Focal outer retinitis associated with optic disc
\end{abstract}




\section{Case Reports in Ophthalmology}

Martínez-Rubio et al.: Paraneoplastic Focal Outer Retinitis

oedema and antibody positivity facilitated the diagnosis of SCLC in a localized stage. SD-OCT allowed the characterization of this entity, expanding the spectrum of paraneoplastic ophthalmological syndromes.

(c) 2020 The Author(s)

Published by S. Karger AG, Basel

\section{Introduction}

Paraneoplastic optic neuropathy (PON) with retinitis is a paraneoplastic syndrome triggered by the production of autoantibodies in patients with carcinoma, especially small cell lung carcinoma (SCLC) [1]. PON has been well documented to occur together with vitritis and neurological symptoms [1], but concurrent retinal involvement is a much less frequent feature of this syndrome. Collapsin response mediator protein (CRMP) 5-IgG is identified as the main autoantibody involved in combined neural and retinal involvement $[2,3]$. The main reason for seeking the prompt identification of this syndrome is the need to exclude the presence of an underlying SCLC [4]. SCLC is a neuroendocrine tumour characterized by an aggressive clinical course with an elusive diagnosis [5]. Indeed, most patients are diagnosed in advanced stages that confer poor survival prognosis. We describe the case of a patient with bilateral optic disc oedema, vitritis and macular lesions whose progressive worsening of ophthalmological and neurological symptoms drove the diagnostic work-up to finally reveal the presence of SCLC. Our aim is to provide a detailed characterization of this acute macular finding and insights into its paraneoplastic aetiology.

\section{Case Report}

A 59-year-old man was referred to the ophthalmology department due to a 3-day history of painless visual loss in his right eye. He also reported asthenia and occasional vomiting over the previous 3 months. He had no relevant medical history apart from a pack-a-day smoking habit for the past 45 years. His best corrected visual acuity was 20/63 and 20/20 (Snellen chart) and a subtle right relative afferent pupillary defect was noted. Visual field assessment revealed a severe diffuse defect in the right eye, while the left visual field was normal. The only pathological finding of the slit lamp examination was an oedematous right optic nerve disc. Neurological examination showed diminished reflexes and mild gait instability, so the patient was admitted to hospital for a complete work-up. Inflammatory markers (C-reactive protein, erythrocyte sedimentation rate) were performed and were normal. An infectious aetiology was excluded by negative serological results for Treponema pallidum, human immunodeficiency virus, hepatitis B virus, hepatitis C virus, Borrelia burgdorferi, Bartonella henselae, Coxiella burnetti, and Toxoplasma gondii. Further, samples were IgG positive but IgM negative for cytomegalovirus, Epstein-Barr virus, and varicella zoster virus. The QuantiFERON-TB Gold test and blood cultures were also negative. Visual evoked potential test showed increased latency in the right eye and magnetic resonance imaging of both the brain and orbits was anodyne. The patient was discharged without a definitive aetiologic diagnosis. During follow-up, visual acuity dropped to hand movement in his right eye and 20/25 in his left eye. A right relative afferent pupillary defect was noted, as well as bilateral optic disc oedema in the fundus examination, with unremarkable macular appearance. Neurologically, the patient had worsening instability and dysmetria of all four limbs, as confirmed by sensory evoked potentials testing and electromyography, hence the patient was admitted again to hospital. The neurological findings, combined with the bilateral optic disc oedema, prompted a lumbar 


\section{Case Reports in Ophthalmology}

Case Rep Ophthalmol 2020;11:507-515

DOI: $10.1159 / 000509861$

(c) 2020 The Author(s). Published by S. Karger AG, Basel www.karger.com/cop

Martínez-Rubio et al.: Paraneoplastic Focal Outer Retinitis

puncture and cerebrospinal fluid (CSF) analysis, this finding an opening pressure of $170 \mathrm{~mm}$ of water, elevated protein content $(137.5 \mathrm{mg} / \mathrm{dL})$ and lymphocyte count $(94 \%)$ but negative cytology and cultures. Additionally, an abnormal chest radiography led to a thoracic CT scan which showed mixed emphysema as well as a right hilar adenopathy with malignant radiological features for which an extended study was advised.

While waiting for the positron emission tomography-computed tomography to be performed, visual acuity worsened to 20/40 in his left eye. Slit lamp exams did not show any presence of anterior chamber cells nor flare. Fundoscopic examinations revealed vitreous haze (RE [+3] > LE [+2]) as per the Standardization of Uveitis Nomenclature classification [6] and creamy-yellow deep retinal lesions showing bilateral macular involvement, distributed in a perifoveal circular pattern in the right eye and a placoid parafoveal lesion in the left eye. Fluorescein angiography (FA) showed papillitis and peripapillary phlebitis (Fig. 1). SD-OCT of the right eye showed a severe disorganization of the outer retinal layers: Henle's fibre layer (HFL) and outer nuclear layer (ONL) revealed focal granular hyperreflectivity with perifoveal coalescence of larger round hyperreflective lesions that transformed into hyperreflective wedge-shaped and then band-like lesions at the fovea. Both the retinal pigment epithelium (RPE) and the ellipsoid segment (ES) layers showed a granular pattern. A greater disorganization of these layers was noted under the hyperreflective lesions in the HFL and ONL. In the fovea, atrophy of the outer retinal layers was noted, with deepening of the foveal depression and, below this, a hyperreflective deposit. Hyperreflective dots in the vitreous cavity corresponding to the vitreous cells were also noted. SD-OCT of the left eye showed vitreous dotted hyperreflectivity and a single hyperreflective tangential band at the fovea, with granular disorganization of outer retinal layers (Fig. 2a-d).

Based on the negative systemic work-up for an infectious aetiology, the anodyne cerebral and orbital MRI and the suspicious right hilar adenopathy, the differential diagnosis included a paraneoplastic syndrome, and hence, serological and CSF onco-neuronal antibodies were tested. Indirect immunofluorescence detected IgG for CRMP5, Hu and amphiphysin, which were confirmed by recombinant western blot analysis both in serum and CSF, this leading to the diagnosis of paraneoplastic syndrome. The patient received intravenous methylprednisolone $1 \mathrm{~g} /$ day for 4 days followed by oral prednisone $30 \mathrm{mg}$ with tapering doses and visual acuity improved to 20/125 and 20/32 in the RE and LE at day 5 of follow-up. Multimodal imaging showed improvement in vitreous haze and shrinkage of the macular lesions. Positron emission tomography-computed tomography showed a hypermetabolic region in the hilum of the right lung. A bronchoscopy endoscopy with ultrasound was performed, and the histopathological analysis of the sample revealed the presence of atypical epithelial cell groups which were positive for CD-56, TTF-1 and synaptophysin, compatible with the diagnosis of a SCLC. According to the American Joint Committee on Cancer TNM staging system, the disease was classified as T3 N2 M0 (Stage IIIA) by our cancer committee. Given the localized malignancy and the patient's good performance status, the patient started a treatment consisting of concomitant chemotherapy (cisplatin and etoposide) and radiotherapy with a curative intent. The patient tolerated the treatment well and the hilar mass decreased from 20 to $13 \mathrm{~mm}$ in diameter, representing a partial response. Prophylactic cranial irradiation was also conducted. Ophthalmologically, a subtenon injection of triamcinolone was given in both eyes while maintaining oral prednisone $10 \mathrm{mg} /$ day. Fundoscopy revealed bilateral persistent vitreous haze $(+1$ and +0.5 , respectively), normalized optic nerves and a progressive fading of the yellowish macular lesions leading to atrophic changes. Right SD-OCT showed a subfoveal collapse of the ONL and ES layers, with granular appearance of the RPE. Interestingly, regression of the left macular hyperreflective band was noted, with suboptimal restoration of the 


\section{Case Reports in Ophthalmology}

Case Rep Ophthalmol 2020;11:507-515

DOI: $10.1159 / 000509861$

c) 2020 The Author(s). Published by S. Karger AG, Basel www.karger.com/cop

Martínez-Rubio et al.: Paraneoplastic Focal Outer Retinitis

outer retinal layers (Fig. 3). In line with this, at 3 months of follow-up, visual acuity in the right eye decreased to $20 / 200$, whereas in the left eye it improved to $20 / 25$.

\section{Discussion}

PON is an uncommon disease, usually diagnosed prior to the diagnosis of an underlying malignancy, most frequently SCLC [1]. PON is characterized by the clinical findings of vitritis and optic disc oedema, frequently in a bilateral presentation [1, 7]. Ocular studies including electrophysiological testing and multimodal retinal imaging, such as FA, fundus autofluorescence (FAF) and OCT offer a detailed characterization of the posterior involvement. However, due to the intense vitritis of our patient, FAF did not add much information. The ocular signs showed by our patient were early oriented towards a paraneoplastic aetiology based on the neurological signs and the suspicious lung mass in a patient with a past history of heavy smoking. However, a complete work-up should exclude infectious, inflammatory, compressive, or neoplastic aetiologies compatible with bilateral vitritis and optic disc oedema. Ancillary testing help in the diagnostic process, where vitreo-retinal biopsy may remain as a final resource specially if a primary intraocular lymphoma is suspected $[1,8]$. Although an infrequent aetiology, primary intraocular lymphoma usually presents as vitritis and optic nerve oedema in the absence of macular oedema. Retinal involvement is rare, but when present, chorioretinal infiltration of lymphoma cells are reported to invade the subretinal pigment space in a preferential pattern [9], imaged as subretinal drusenoid deposits with infrared imaging and SDOCT [10]. Orbital and cerebral neuroimaging are mandatory in order to rule out any enhancement of the central nervous system that may guide diagnosis depending on the affected location, for example, a metastatic infiltration of the optic nerve, retrobulbar optic neuritis or central nervous system lymphoma. In the concrete case of PON, a thorough neurological examination is highly recommended as this entity frequently coexists with encephalomyeloradiculoneuropathy $[1,11]$.

Few publications have described retinal abnormalities in the setting of PON. Saito et al. [2] reported 1 patient with SCLC with bilateral PON and CRMP5-IgG, recoverin and enolase serum positivity in whom OCT showed bilateral neuroretinitis, with bilateral subretinal detachment extending from both swollen optic nerve discs. Additionally, the right macula of the patient had a focal hyperreflective dome-shaped lesion extending from the outer border of the inner nuclear layer to the RPE with subsequent disorganization of all the layers involved. In 2012, Casswell et al. [3] reported the case of a patient diagnosed with bilateral PON secondary to SCLC and anti-CRMP5, anti-amphiphysin and anti-Hu serum positivity, and bilateral focal external atrophy of the macula.

To the best of our knowledge, our case represents the first report of PON with bilateral retinopathy in which macular abnormalities have been documented in their initial stages by SD-OCT. In the right macula, multifocal hyperreflective foci were observed, in what we believe were different stages of the same process: they arose at the ONL and HFL, increased in size, and transformed into hyperreflective wedge-shaped lesions that extended to the limit of the RPE. This phenomenon was best identified in the foveal area, with a hyperreflective placoid lesion, which showed thinning and atrophy at its edges. There were multiple spots at the HFL, ONL and ES layers which were already atrophic or starting to collapse. The RPE and junctional layers showed a mottled appearance across the macular scans. In the left eye, a wedge-shaped hyperreflective lesion was identified at the fovea, emerging from the inner border of the HFL and masking the normal reflectivity of the retinal layers underneath. RPE mottling and 


\section{Case Reports in Ophthalmology}

Martínez-Rubio et al.: Paraneoplastic Focal Outer Retinitis

atrophy were observed, as were vitreous hyperreflective dots. The atrophic changes described in the patients in Saito and Casswell's reports are compatible with the advanced stage of our patient's right maculopathy $[2,3]$.

Our patient tested positive for the onconeural antibodies anti-CRMP5, anti-Hu and antiamphiphysin. CRMP5-IgG is the main antibody reported in PON with underlying SCLC $[4,12]$. Anti-Hu and anti-amphiphysin are well characterized antibodies in paraneoplastic neurological syndromes $[4,12,13]$ and have also been reported in PON $[3,7,12]$. In contrast to the proposed mechanism of photoreceptor apoptosis in anti-recoverin cancer-associated retinopathy $[13,14]$, the pathogenic pathways of anti-CRMP5, anti-Hu and anti-amphiphysin in PON and retinitis are not fully understood. The suggested mechanisms of pathogenesis are a directly autoantibody-mediated photoreceptor loss or an indirect activation of immune responses that eventually lead to photoreceptor cell loss [14]. We hypothesize that these pathogenic mechanisms might be responsible for the retinal abnormalities seen in our patient.

In conclusion, we describe a case of PON with acute focal outer retinitis associated with SCLC. It represents a rare paraneoplastic retinal entity that differs clinically from cancer-associated retinopathy, cancer-associated cone dysfunction and paraneoplastic vitelliform retinopathy $[12,13]$. Focal outer retinitis was identified as acute focal central involvement, visible as yellowish deep foveal lesions in fundoscopy. SD-OCT revealed the following features: (1) hyperreflective foci emerging from the HFL and ONL and transforming into (2) hyperreflective wedges that involved the outer retinal layers and RPE layer; (3) a granular hyperreflective pattern on the RPE; and (4) collapse of the most severely affected foveal outer retinal layers leading to atrophy. The lesion in the left fovea partially recovered after treatment. In spite of the pathway being poorly understood, the precise description of the different phenotypes of paraneoplastic syndromes may help ophthalmologists in the search for an underlying malignancy responsible for the syndrome, as well as guide treatment of the macular manifestations. SCLC has an elusive diagnosis with the majority of patients being diagnosed in an advanced, metastatic stage [5] that implies a median overall survival of less than 10 months [15]. Paraneoplastic syndromes may serve as markers for earlier diagnosis in localized stages, thus ameliorating the general prognosis of these patients.

\section{Statement of Ethics}

The authors confirm that the patient has given his written informed consent to publish his case report (including publication of images).

\section{Conflict of Interest Statement}

The authors have no conflicts of interest to declare.

\section{Funding Sources}

The authors do not have any funding sources to declare. 


\section{Case Reports in Ophthalmology}

\begin{tabular}{l|l}
\hline DOI: $10.1159 / 000509861$ & (c) 2020 The Author(s). Published by S. Karger AG, Basel
\end{tabular} www.karger.com/cop

Martínez-Rubio et al.: Paraneoplastic Focal Outer Retinitis

\section{Author Contributions}

The authors declare their contribution in the present manuscript as specified below:

Clara Martínez-Rubio: Concept and design, data acquisition, data analysis and interpretation, drafting manuscript, critical revision of manuscript, technical support, supervision, final approval for publication.

Lena Giralt: Concept and design, data acquisition, data analysis and interpretation, drafting manuscript, critical revision of manuscript, technical support, supervision, final approval for publication.

Inazio Lacambra: Concept and design, data acquisition, data analysis and interpretation, drafting manuscript, critical revision, technical support, final approval for publication.

Joseba Artaraz: Concept and design, data acquisition, data analysis and interpretation, drafting manuscript, critical revision, technical support, final approval for publication.

Alex Fonollosa: Concept and design, data acquisition, data analysis and interpretation, drafting manuscript, critical revision of manuscript, technical support, supervision, final approval for publication.

\section{References}

1 Cohen DA, Bhatti MT, Pulido JS, Lennon VA, Dubey D, Flanagan EP, et al. Collapsin response-mediator protein 5-associated retinitis, vitritis, and optic disc edema. Ophthalmology. 2020 Feb;127(2):221-9.

2 Saito M, Saito W, Kanda A, Ohguro H, Ishida S. A case of paraneoplastic optic neuropathy and outer retinitis positive for autoantibodies against collapsin response mediator protein-5, recoverin, and $\alpha$-enolase. BMC Ophthalmol. 2014 Jan;14(1):5.

3 Casswell EJ, Pringle E, Thuang C, Sanders MD, Graham EM. Clinical and Histological Features of Small Cell Lung Cancer Paraneoplastic Inflammatory Uveitis. Ocul Immunol Inflamm. 2016 Oct;24(5):503-7.

4 Pittock SJ, Kryzer TJ, Lennon VA. Paraneoplastic antibodies coexist and predict cancer, not neurological syndrome. Ann Neurol. 2004 Nov;56(5):715-9.

5 Früh M, De Ruysscher D, Popat S, Crinò L, Peters S, Felip E; ESMO Guidelines Working Group. Small-cell lung cancer (SCLC): ESMO Clinical Practice Guidelines for diagnosis, treatment and follow-up. Ann Oncol. 2013 Oct;24 Suppl 6:vi99-105.

6 Jabs DA, Nussenblatt RB, Rosenbaum JT; Standardization of Uveitis Nomenclature (SUN) Working Group. Standardization of uveitis nomenclature for reporting clinical data. Results of the First International Workshop. Am J Ophthalmol. 2005 Sep;140(3):509-16.

7 Cross SA, Salomao DR, Parisi JE, Kryzer TJ, Bradley EA, Mines JA, et al. Paraneoplastic autoimmune optic neuritis with retinitis defined by CRMP-5-IgG. Ann Neurol. 2003 Jul;54(1):38-50.

8 Rahimy E, Sarraf D. Paraneoplastic and non-paraneoplastic retinopathy and optic neuropathy: evaluation and management [Internet]. Surv Ophthalmol. 2013 Sep-Oct;58(5):430-58.

9 Dean JM, Novak MA, Chan CC, Green WR. Tumor detachments of the retinal pigment epithelium in ocular/ central nervous system lymphoma. Retina. 1996;16(1):47-56.

10 Campbell JP, Davis S, Flaxel C, Rosenbaum JT, Lin P. Acquired drusenoid deposits on infrared imaging as a sign of vitreoretinal lymphoma. Ocul Immunol Inflamm. 2014 Apr;22(2):161-3.

11 Bataller L, Dalmau J. Neuro-ophthalmology and paraneoplastic syndromes. Curr Opin Neurol. 2004 Feb;17(1):3-8.

12 Dubey D, Jitprapaikulsan J, Bi H, Do Campo RV, McKeon A, Pittock SJ, et al. Amphiphysin-IgG autoimmune neuropathy: A recognizable clinicopathologic syndrome. Neurology. 2019 Nov;93(20):e1873-80.

13 Graus F, Delattre JY, Antoine JC, Dalmau J, Giometto B, Grisold W, et al. Recommended diagnostic criteria for paraneoplastic neurological syndromes. J Neurol Neurosurg Psychiatry. 2004 Aug;75(8):1135-40.

14 Adamus G, Brown L, Schiffman J, Iannaccone A. Diversity in autoimmunity against retinal, neuronal, and axonal antigens in acquired neuro-retinopathy. J Ophthalmic Inflamm Infect. 2011 Sep;1(3):111-21.

15 Foster NR, Qi Y, Shi Q, Krook JE, Kugler JW, Jett JR, et al. Tumor response and progression-free survival as potential surrogate endpoints for overall survival in extensive stage small-cell lung cancer: findings on the basis of North Central Cancer Treatment Group trials. Cancer. 2011 Mar;117(6):1262-71. 
Case Reports in Ophthalmology
Case Rep Ophthalmol 2020;11:507-515

DOI: 10.1159/000509861

(c) 2020 The Author(s). Published by S. Karger AG, Basel www.karger.com/cop

Martínez-Rubio et al.: Paraneoplastic Focal Outer Retinitis
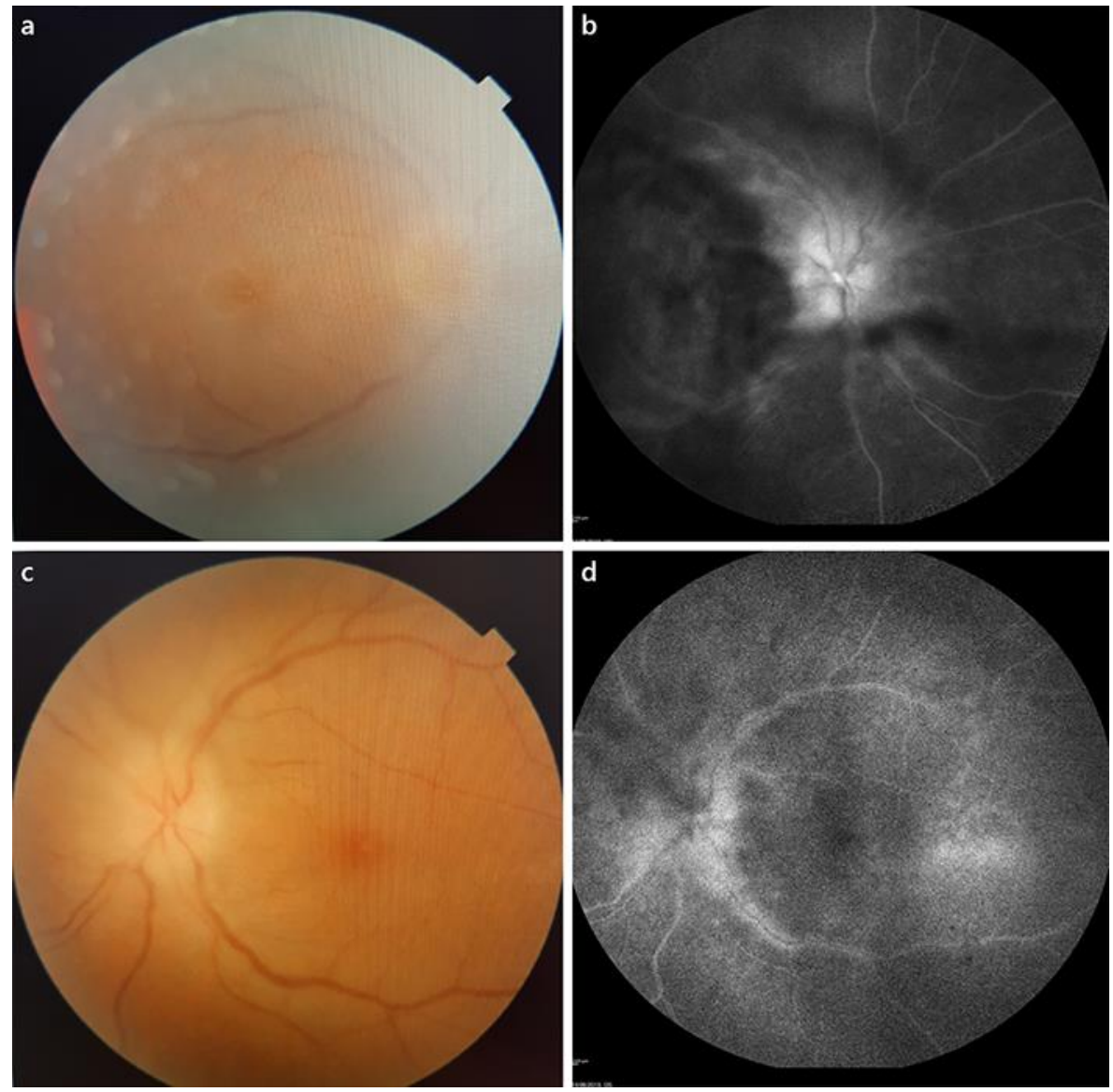

Fig. 1. Ophthalmologic exam 2 weeks after onset. a, b Vitreous haze hindered fundus examination in both eyes; bilateral optic disc oedema is noted as well as yellowish foveal lesions. c, d Fluorescein angiography reveals hyperfluorescence of both optic nerve discs as well as hyperfluorescent staining of peripapillary venous walls. 


\section{Case Reports in Ophthalmology}
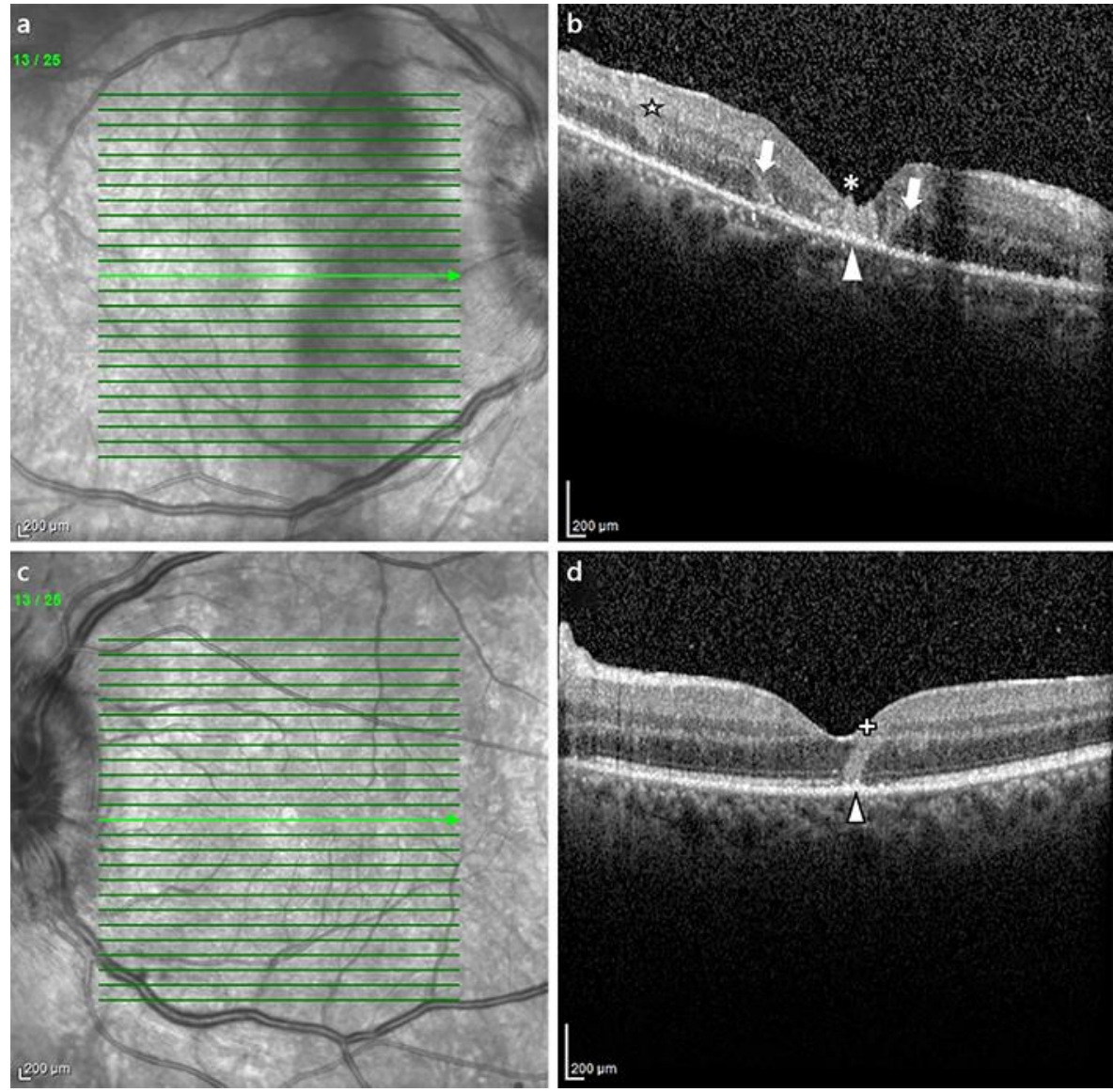

Fig. 2. SD-OCT follow-up. RE, right eye. LE, left eye. HFL, Henle's fibre layer. ONL, outer nuclear layer. ES, external segments. RPE, retinal pigment epithelium. a, b Near-infrared imaging at 2 weeks of follow-up. Prominent vitreous haze in RE and optic disc oedema are noted. c (B-Scan SD-OCT, RE at 2 weeks) In the fovea, a hyperreflective lesion is identified $(*)$, with atrophy of the outer retinal layers (limits are identified by arrows). Hyperreflective conglomerates appear in the inner border of the outer nuclear layer ( and ES layers show a granular appearance (arrowhead). Hyperreflective dots in the vitreous and choroid can be identified. $\mathbf{d}$ (B-Scan SD-OCT, LE at 2 weeks) A hyperreflective wedge comprising the inner border of the outer nuclear layer traverses the outer foveal layers to reach the RPE layer ("+"), where a granular hyperreflectivity can be seen (arrowhead). 


\section{Case Reports in Ophthalmology}
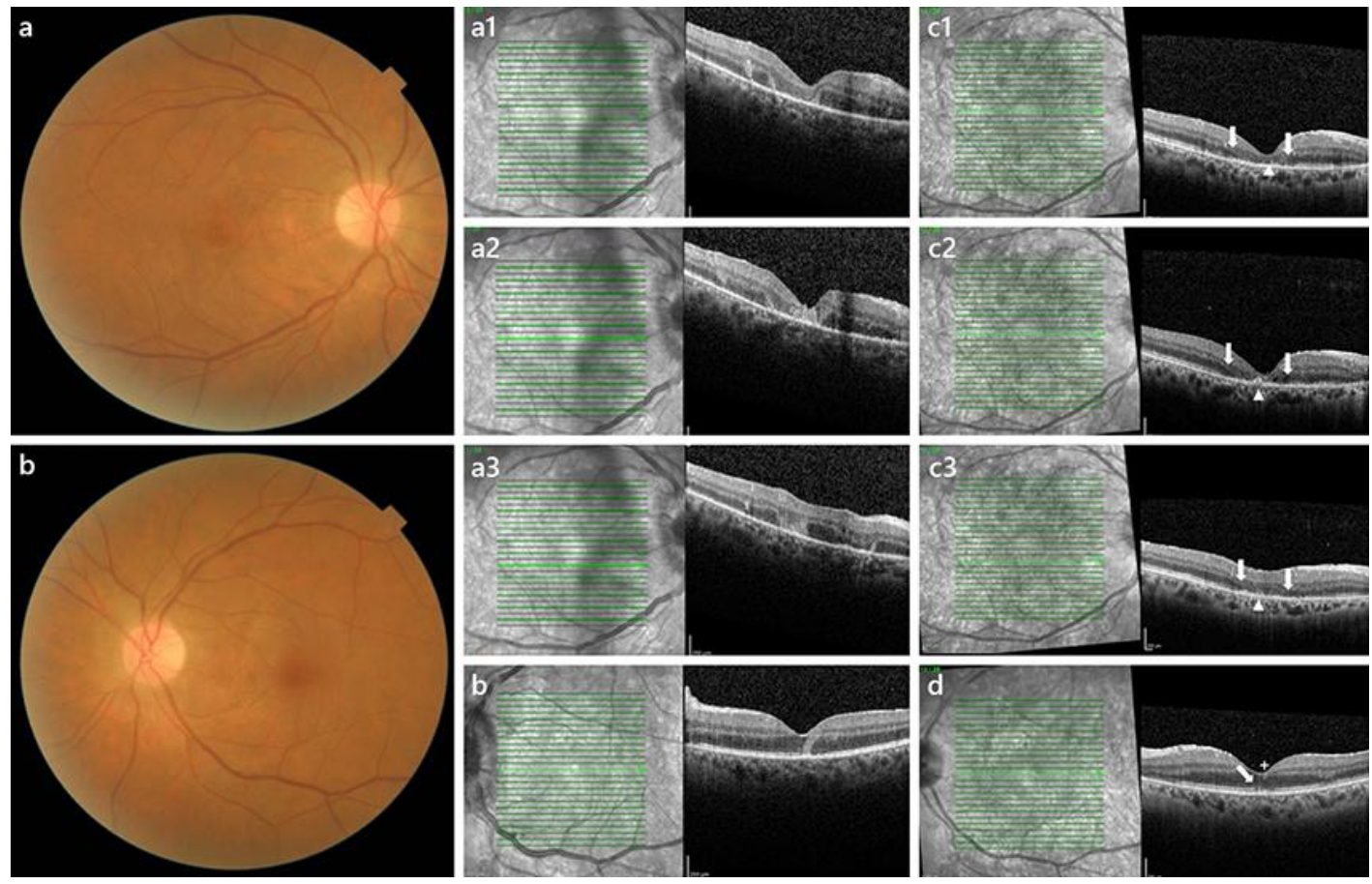

Fig. 3. Ophthalmological examination at last visit. HFL, Henle's fibre layer. ONL, outer nuclear layer. ES, external segments. RPE, retinal pigment epithelium. a, b Retinography of the right eye (a) and left eye (b) at the last visit: noticeable reduction in the vitreous haze, resolution of the optic disc oedema with subtle pallor and yellowish dotted macular appearance. a1, a2, a3 SD-OCT of the right eye at 2 weeks. c1, c2, c3 SD-OCT of the right eye at 2 months: foveal atrophy of the HFL, ONL and ES layer (limits are identified by arrows). Degenerative cysts in the perifoveal outer nuclear layer can be identified in the middle row scan (c2). A residual granular hyperreflective appearance of the RPE layer is noted in all three scans (arrowheads) (c1, c2, c3). b Optical coherence tomography of left eye at 2 weeks. $\mathbf{d}$ Optical coherence tomography of the left eye at 2 months: a partial granular disruption of the ES layer (arrow) and remaining hyperreflective changes in the RPE and inner border of the ONL ("+"). 